\title{
Clinical features of autosomal recessive polycystic kidney disease in the Japanese population and analysis of splicing in PKHD1 gene for determination of phenotypes
}

\author{
Shinya Ishiko ${ }^{1} \cdot$ Naoya Morisada $^{1,2}$ D $\cdot$ Atsushi Kondo ${ }^{1} \cdot$ Sadayuki Nagai $^{1} \cdot$ Yuya Aoto $^{1} \cdot$ Eri Okada $^{1} \cdot$ Rini Rossanti $^{1}$. \\ Nana Sakakibara ${ }^{1}$. China Nagano ${ }^{1} \cdot$ Tomoko Horinouchi $^{1} \cdot$ Tomohiko Yamamura $^{1} \cdot$ Takeshi Ninchoji $^{1}$. \\ Hiroshi Kaito ${ }^{3}$ Riku Hamada ${ }^{4}$ Yuko Shima ${ }^{5}$. Koichi Nakanishi ${ }^{6}$. Masafumi Matsuo ${ }^{7}$ Kazumoto lijima ${ }^{1}$. \\ Kandai Nozu ${ }^{1}$
}

Received: 18 June 2021 / Accepted: 7 September 2021 / Published online: 18 September 2021

(c) The Author(s) 2021

\begin{abstract}
Background Autosomal recessive polycystic kidney disease (ARPKD) is caused by mutations in the PKHD1 gene. The clinical spectrum is often more variable than previously considered. We aimed to analyze the clinical features of genetically diagnosed ARPKD in the Japanese population.

Methods We conducted a genetic analysis of patients with clinically diagnosed or suspected ARPKD in Japan. Moreover, we performed a minigene assay to elucidate the mechanisms that could affect phenotypes.

Results $P K H D 1$ pathogenic variants were identified in 32 patients (0-46 years). Approximately one-third of the patients showed prenatal anomalies, and five patients died within one year after birth. Other manifestations were detected as follows: chronic kidney disease stages 1-2 in 15/26 (57.7\%), Caroli disease in 9/32 (28.1\%), hepatic fibrosis in 7/32 (21.9\%), systemic hypertension in 13/27 (48.1\%), and congenital hypothyroidism in 3 patients. There have been reported that truncating mutations in both alleles led to severe phenotypes with perinatal demise. However, one patient without a missense mutation survived the neonatal period. In the minigene assay, c.2713C $>\mathrm{T}$ (p.Gln905Ter) and c.6808 + $1 \mathrm{G}>\mathrm{A}$ expressed a transcript that skipped exon 25 (123 bp) and exon 41 (126 bp), resulting in an in-frame mutation, which might have contributed to the milder phenotype. Missense mutations in cases of neonatal demise did not show splicing abnormalities.

Conclusion Clinical manifestations ranged from cases of neonatal demise to those diagnosed in adulthood. The minigene assay results indicate the importance of functional analysis, and call into question the fundamental belief that at least one non-truncating mutation is necessary for perinatal survival.
\end{abstract}

Keywords Autosomal recessive polycystic kidney disease $\cdot$ PKHDl $\cdot$ Hepatic fibrosis $\cdot$ Congenital hypothyroidism · Minigene assay

Naoya Morisada

morisada@med.kobe-u.ac.jp

1 Department of Pediatrics, Kobe University Graduate School of Medicine, 7-5-1, Kusunoki-cho, Chuo-ku, Kobe, Hyogo 650-0017, Japan

2 Department of Clinical Genetics, Hyogo Prefectural Kobe Children's Hospital, 1-6-7, Minatojimaminami-machi, Chou-ku, Kobe, Hyogo 650-0047, Japan

3 Department of Nephrology, Hyogo Prefectural Kobe Children's Hospital, 1-6-7, Minatojimaminami-machi, Chou-ku, Kobe, Hyogo 650-0047, Japan

4 Department of Nephrology, Tokyo Metropolitan Children's Medical Center, 2-8-29 Musashidai, Fichu-shi, Tokyo 183-8561, Japan
Department of Pediatrics, Wakayama Medical University, 811-1, Kimiidera, Wakayama, Wakayama 641-8509, Japan

6 Department of Child Health and Welfare (Pediatrics), Graduate School of Medicine, University of Ryukyus, 207 Uehara, Nishihara-cho, Nakagami-gun, Okinawa 903-2015, Japan

7 KNC Department of Nucleic Acid Drug Discovery, Faculty of Rehabilitation, Kobe Gakuin University, 518 Arise Ikawadani-cho, Nishi-ku, Kobe Hyogo 651-2113, Japan 


\section{Introduction}

Autosomal recessive polycystic kidney disease (ARPKD) is an inherited cilia-related disorder characterized by the association of bilateral renal cystic disease and congenital hepatic fibrosis. The polycystic kidney and hepatic disease 1 (PKHD1) gene has been identified as the causative gene for ARPKD [1, 2], with 590 types of pathogenic mutation reported in The Human Gene Mutation Database to date (http://www.hgmd.cf.ac.uk, HGMD). PKHD1, extending over a genomic segment of at least $470 \mathrm{~kb}$ on chromosome $6 \mathrm{p} 12$, is one of the largest disease-causing genes in the human genome. The longest PKHDI transcript contains 67 exons that encodes a protein comprising 4074 amino acids. PKHD 1 encodes a single-transmembrane protein, called polyductin/fibrocystin, which is mainly expressed in the kidneys and liver. Fibrocystin localizes in the ciliary membrane, and it may be involved in regulating cell to cell adhesion and proliferation; it also acts as a membrane-bound receptor [1-3]. However, the detailed function of $P K H D 1$ and the onset mechanism of ARPKD are still unknown. Recently, mutations in $D A Z$ interacting protein 1-like (DZIPIL) have been reported in patients with ARPKD, suggesting that ARPKD is not a homogeneous disorder and DZIPIL may also be involved in its pathogenesis [4], however, whether there is a causative relationship between DZIPIL and ARPKD requires further investigation [5].

The clinical spectrum of the disease is often more variable than previously considered [6-8]. Most cases have been identified either in utero or at birth. Approximately $30-50 \%$ of affected neonates die shortly after birth due to severe pulmonary hypoplasia and secondary respiratory insufficiency $[9,10]$. In contrast, those who survive through the perinatal period express variable disease phenotypes, and some elderly patients with ARPKD are only moderately affected $[8,9]$. A genotype-phenotype correlation has been reported in which patients with biallelic truncating mutations in PKHD1 show a severe phenotype with perinatal demise, while children surviving the postnatal period carry at least one missense mutation [9]. However, some patients with missense mutations can present with a phenotype that is as severe as that associated with truncating mutations, suggesting that complex transcriptional alterations may play a role in defining the phenotype [11].

To date, no multi-center study has described the genotype and phenotype of genetically diagnosed ARPKD patients in Japanese populations. Therefore, we aimed to analyze the clinical features of patients who were referred to our institute for gene testing and genetically diagnosed with ARPKD. Additionally, we conducted a functional analysis using a minigene assay to reveal the existence of aberrant splicing caused by six mutations detected in our study to further investigate the genotype-phenotype correlation. One patient without a missense mutation survived the neonatal period, and this result was contradictory to previous reports. Thus, we aimed to elucidate the underlying mechanism that led to the milder phenotype despite the absence of a missense mutation. Additionally, three patients with one missense mutation died soon after birth. We aimed to evaluate whether these missense mutations affected splicing.

\section{Materials and methods}

\section{Study design}

We conducted gene testing using next-generation sequencing (NGS) in patients with clinically diagnosed or suspected ARPKD at Japanese hospitals between April 2016 and April 2021. We analyzed cases in which two or more PKHDI or $D Z I P I L$ variants were considered pathogenic. Detailed information regarding clinical features was obtained from the referring clinician or hospital records of patients.

\section{Genetic analysis}

DNA was isolated from peripheral blood samples using a QuickGene Mini 80 system (Wako Pure Chemical Industries, Ltd., Tokyo, Japan) according to the manufacturer's instructions. Direct sequencing or targeted sequencing using NGS was performed on the genes responsible for inherited renal diseases. For NGS, we used a HaloPlex HS or SureSelect (Agilent Technologies, Santa Clara, CA, USA) according to the manufacturer's instructions, and sequencing was performed using the MiSeq platform (Illumina, San Diego, CA, USA). HaloPlex HS was used for targeted sequencing of 128 (version 2, Supplementary Table 1), 172 (version 4, Supplementary Table 2), 159 (version 5, Supplementary Table 3), 164 (version 6, Supplementary Table 4), and 181 (version 7, Supplementary Table 5) genes, and SureSelect was used for the targeted sequencing of 203 (version 8, Supplementary Table 6) and 193 genes (version 9, Supplementary Table 7) associated with congenital anomalies of the kidney and urinary tract, and various cystic kidney diseases, including ARPKD, autosomal dominant polycystic kidney disease, and nephronophthisis, as cataloged in OMIM (https://www.omim.org) or PubMed (https://pubmed.ncbi. nlm.nih.gov) database.

Data were analyzed using SureCall 4.0 (Agilent Technologies), a software for end-to-end NGS data analysis. The cDNA reference numbers of PKHDI and DZIPIL 
were NM_138694.3 and 173,543.2, respectively. Pathogenicity predictions were performed in accordance with the guidelines of the American College of Medical Genetics (Supplementary Table 8). Several websites, including CADD (https://cadd.gs.wa shington.edu/), PROVEAN (http://provean.jcvi.org/index. php), SIFT (https://sift. bii.a-star.edu.sg/), PolyPhen-2 (http://genetics.bwh. harvard.edu/pph2/), and Mutation Taster (http://www. pathogenic varianttaster.org/) were used to predict variant pathogenicity (Supplementary Table 9). The splice sites of each variant were predicted using Human Splicing Finder (https://hsf.genomnis.com/home). Pair analysis using SureCall was used to determine the changes in copy number relative to a reference [12]. Changes in copy number were confirmed by multiplex ligation and probe amplification (MLPA) using SALSA P341-B4/P342-C1 PKHD1 (MRC-Holland, Amsterdam, the Netherlands), as suggested by the manufacturer. The MLPA test was performed twice to confirm abnormal changes.

\section{Minigene assay}

We conducted in vitro analysis using a minigene assay for the following: missense mutations, c. $9533 \mathrm{G}>\mathrm{T}$ (p.Gly3178Val) in SC293, c.3944 T > G (p.Leu1315Arg) in SC324, and c.983G > A (p.Arg328Gln) in SC589; splice site mutations, c.8555-2A $>\mathrm{C}$ in $\mathrm{SC} 324$ and c. $6808+1 \mathrm{G}>\mathrm{A}$ in SC499; and nonsense mutation, c. 2713C > T(p.Gln905Ter) in SC499. To create hybrid minigene constructs, we used the previously developed H492 vector, which is based on the pcDNA 3.0 mammalian expression vector (Invitrogen, Carlsbad, CA, USA) [13]. We cloned DNA fragments from both wild-type and patient peripheral leukocytes containing exons and introns around the target variants in $P K H D 1$ gene using In-Fusion cloning methods with the HD Cloning Kit (Takara Bio Inc., Kusatsu, Japan) according to manufacturer's instructions (Supplementary Fig. 1). Primers used for cloning in the minigene assay for each mutation are listed in Supplementary Table 10. The hybrid minigenes were confirmed by sequencing, and they were transfected into HEK293T cells using Lipofectamine ${ }^{\circledR} 2000$ (Thermo Fisher Scientific, Waltham, MA, USA). Total RNA was extracted from cells after $24 \mathrm{~h}$ using the RNeasy Plus Mini Kit (QIAGEN, Hilden, Germany). Total RNA was reverse-transcribed using ReverTra Ace (Toyobo, Osaka, Japan). PCR was performed using a forward primer corresponding to a segment upstream of exon A and reverse primer complementary to a segment downstream of exon $\mathrm{B}$, as previously described. PCR products were analyzed via electrophoresis on a $1.5 \%$ agarose gel using a DNA ladder, and this was followed by direct sequencing.

\section{Results}

\section{Patient characteristics}

PKHD1 pathogenic variants were identified in 32 patients from 31 families. The DZIPIL mutation was not detected in any patient. There were 9 men and 23 women, and the median age of patients at the time of gene testing was 5 years (0-46 years) (Table 1$)$. The patients were recruited from 26 hospitals in Japan.

\section{PKHD1 mutations}

In total, 64 mutations were identified. SC481 and her elder brother harbored the same mutations, and two patients (SC746 and SC756) had homozygous mutations; thus, we analyzed 60 variants, of which, 58 were detected via NGS and confirmed via direct sequencing, and two were detected via MLPA. Among the 60 variants, 34 missense mutations (56.7\%), 17 nonsense mutations (28.3\%), four disruption of a conserved splice site $(6.7 \%)$, three frameshift mutations (5.0\%), and two large deletions (3.3\%) were detected. Among the point mutations, 20 variants were novel mutations in HGMD, dbSNP, and ClinVar (Table 2). c.5174G > C (p.Trp1725Ser), c.6794A > T (p.His2265Leu), c.7867delT (p.Try2623Thrfs*44), and c.9533G $>$ T (p.Gly3178Val) genes were detected in multiple patients (Table 2, Supplementary Table 9).

\section{Clinical features}

Ten of the 32 patients (31.3\%) showed prenatal anomalies with oligohydramnios $(n=9 / 10)$, kidney enlargement $(n=5 / 10)$, cystic kidney $(n=3 / 10)$, or increased renal echogenicity $(n=3 / 10)$. Three of these patients (SC293, SC324, and SC589) died soon after birth due to respiratory failure, and two patients (SC697 and SC746) died within the first year of life. Two patients were suspected to have ARPKD soon after birth following the detection of respiratory failure and enlarged kidneys. The remaining patients were primarily diagnosed or suspected incidentally after the neonatal period, especially through medical checkups for infants or at school $(n=3)$, or workplace $(n=3)$ and school urinary screening $(n=2)$. Another reason for the initial visit to doctors was urinary tract infection $(n=2)$. The most common manifestation leading to diagnosis in pediatric cases was enlarged kidneys while in all the four adult cases, kidney dysfunction led to a diagnosis. The occasions for consultation and manifestations are presented in Table 2.

More than half of the patients $(n=15 / 26)$ at the analyzed visit showed native kidney function in chronic kidney 
Table 1 Patient characteristics

\begin{tabular}{|c|c|c|c|c|}
\hline & Patients $(n=32)$ & $\mathrm{T} / \mathrm{T}$ & $\mathrm{T} / \mathrm{NT}$ & NT/NT \\
\hline \multicolumn{5}{|l|}{ Age at suspected diagnosis } \\
\hline Median & 4 months & & & \\
\hline Range & GA $25 w-36$ years & & & \\
\hline \multicolumn{5}{|l|}{ Age at genetic diagnosis } \\
\hline Median & 5 years & & & \\
\hline Range & 0 day -46 years & & & \\
\hline \multicolumn{5}{|l|}{ Gender } \\
\hline Male & 9 & 1 & 5 & 3 \\
\hline Female & 23 & 2 & 15 & 6 \\
\hline \multicolumn{5}{|l|}{ Kidney function* } \\
\hline CKD stage 1 & $9 / 26(34.6 \%)$ & & 5 & 4 \\
\hline CKD stage 2 & $6 / 26(23.1 \%)$ & & 4 & 2 \\
\hline CKD stage 3 & $5 / 26(19.2 \%)$ & & 5 & \\
\hline CKD stage 4 & $2 / 26(7.7 \%)$ & & 2 & \\
\hline CKD stage 5 & $0 / 26(0 \%)$ & & & \\
\hline Renal replacement therapy & $4 / 26(15.44 \%)$ & 2 & & 2 \\
\hline \multicolumn{5}{|l|}{ Hepatic disease $^{\mathrm{a}}$} \\
\hline Caroli disease & $9 / 32(28.1 \%)$ & 2 & 5 & 2 \\
\hline Hepatic fibrosis & $7 / 32(21.9 \%)$ & & 3 & 4 \\
\hline Hepatic cysts & $2 / 32(6.2 \%)$ & & 1 & 1 \\
\hline \multicolumn{5}{|l|}{ Other manifestations $\mathrm{s}^{\mathrm{a}}$} \\
\hline Hypertension (children) & $12 / 23$ & 2 & 7 & 3 \\
\hline Hypertension (adult) & $1 / 4$ & & 1 & \\
\hline Respiratory failure at birth & 7 & 2 & 4 & 1 \\
\hline Urinary tract infection & 4 & & 4 & \\
\hline Congenital hypothyroidism & 3 & & 1 & 2 \\
\hline Urolithiasis & 2 & & & 2 \\
\hline Thrombocytopenia & 1 & & & 1 \\
\hline Splenomegaly & 1 & & & 1 \\
\hline Vesicoureteral reflux & 1 & & 1 & \\
\hline Perthes disease, inguinal hernia & 1 & & & 1 \\
\hline
\end{tabular}

$C K D$ chronic kidney disease, $G A$ gestational age, $N T$ non-truncating mutation, $T$ truncating mutation, $w$ weeks

${ }^{a}$ Only the number of evaluable patients is shown

disease (CKD) stage 1 or 2 . Four patients underwent peritoneal dialysis, and three of them were required to undergo hemodialysis during the neonatal period. None of the patients underwent kidney transplantation at the time of genetic analysis. The distribution between patients' age and kidney functions, classified by the mutation type, is shown in Fig. 1.

Among the 27 surviving cases, imaging findings via ultrasonography and/or CT scan detected Caroli disease in 8 of 27 patients (29.6\%) and hepatic fibrosis in 6 patients $(22.2 \%)$. One patient underwent liver transplantation at the age of 14 years (SC619). Thrombocytopenia and splenomegaly due to portal hypertension were detected in one patient (SC272). Almost half of the pediatric patients $(n=12 / 23)$ showed systemic hypertension while one of the four adult patients did. Congenital hypothyroidism was observed in three patients in the neonatal mass-screening test, and treatment with levothyroxine was required.

\section{Genotype-phenotype correlation in PKHD1}

Nine patients harbored missense mutations on both alleles, and all of them survived the neonatal period, although one patient (SC746) died at the age of 5 months. Additionally, 21 patients had one missense mutation, and three of them died during the neonatal period due to respiratory failure (SC293, SC324, and SC589). Three patients had no missense mutations (SC432, SC499, and SC697), and all patients were diagnosed at an early stage in their life (Fig. 1). 


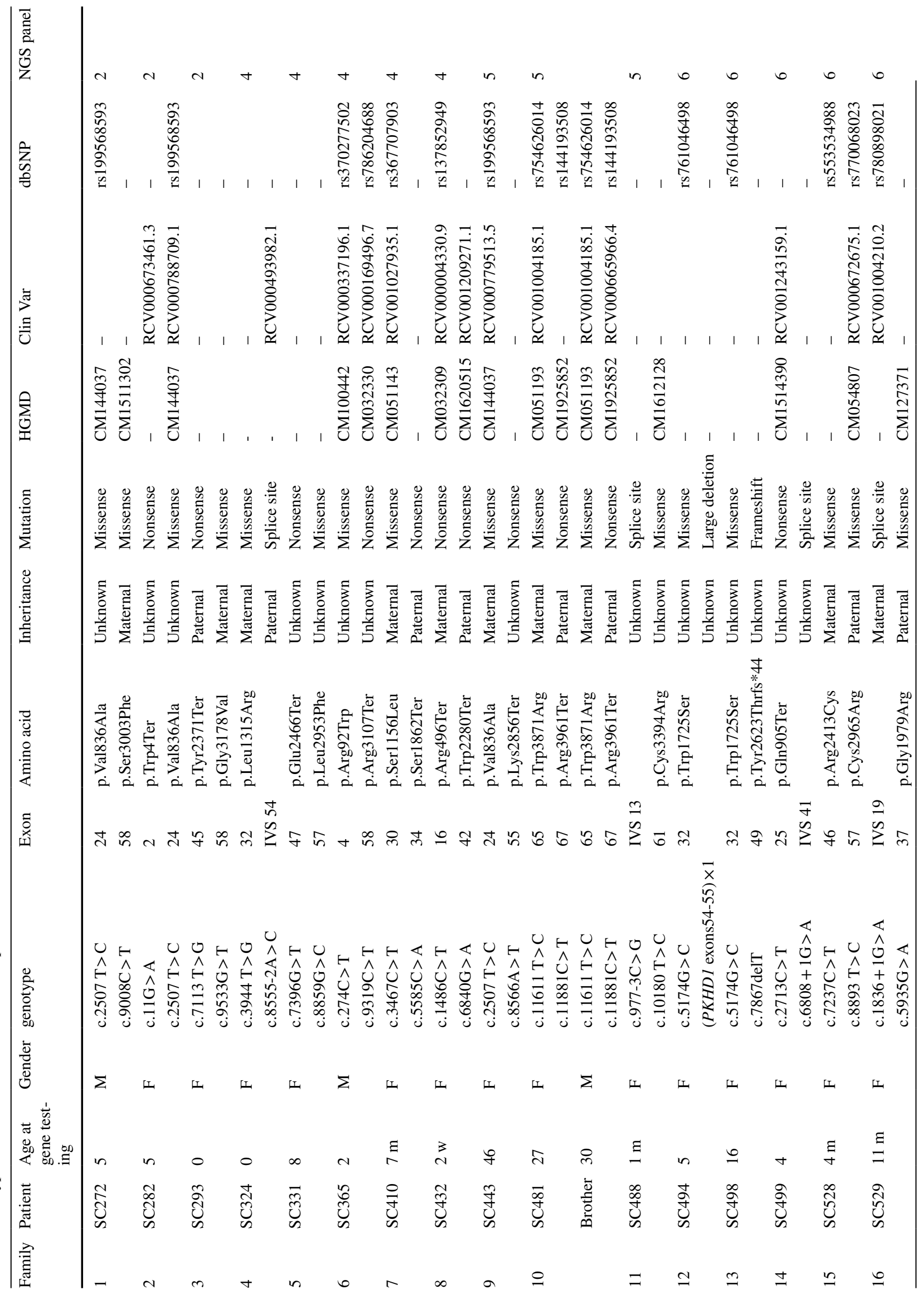




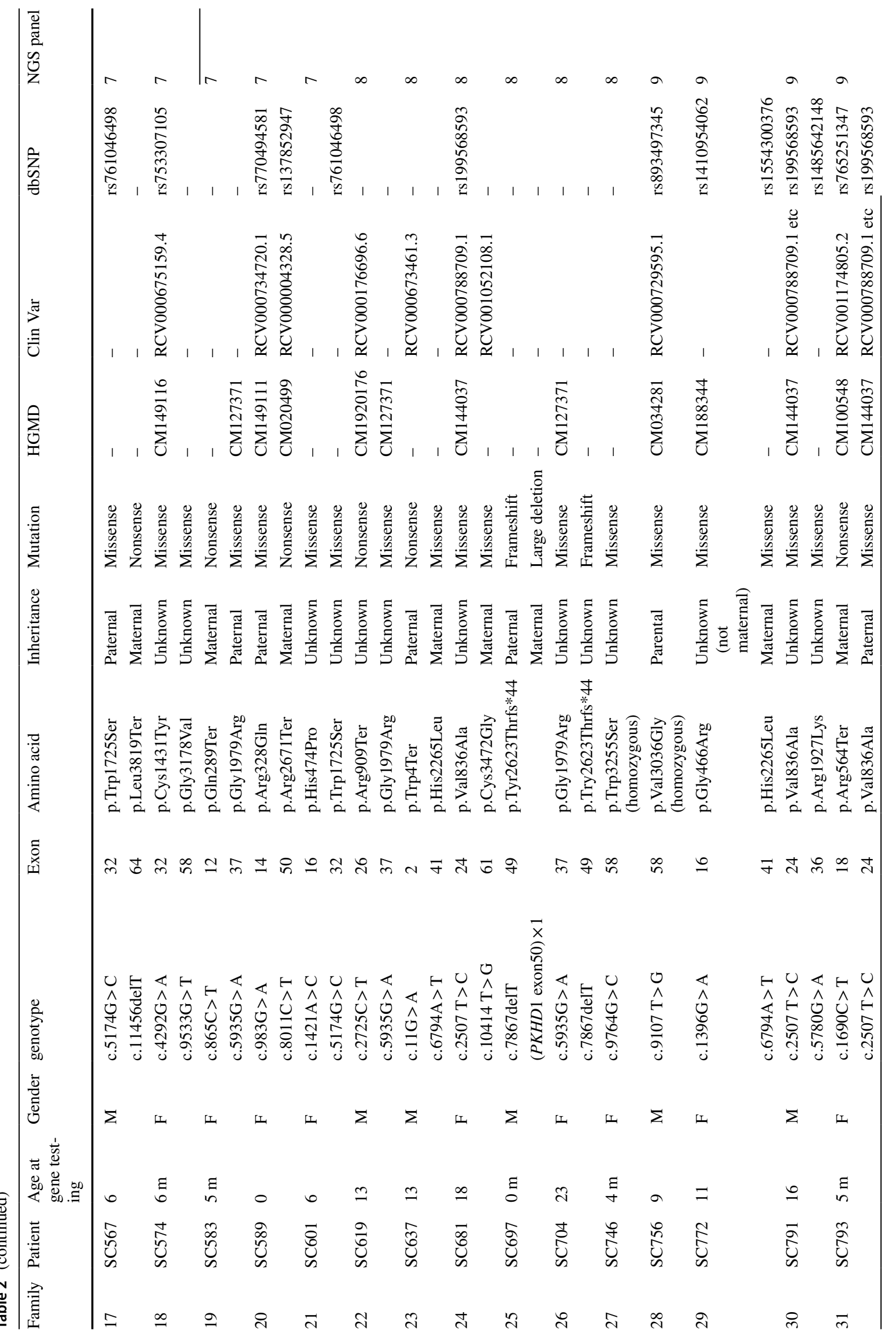




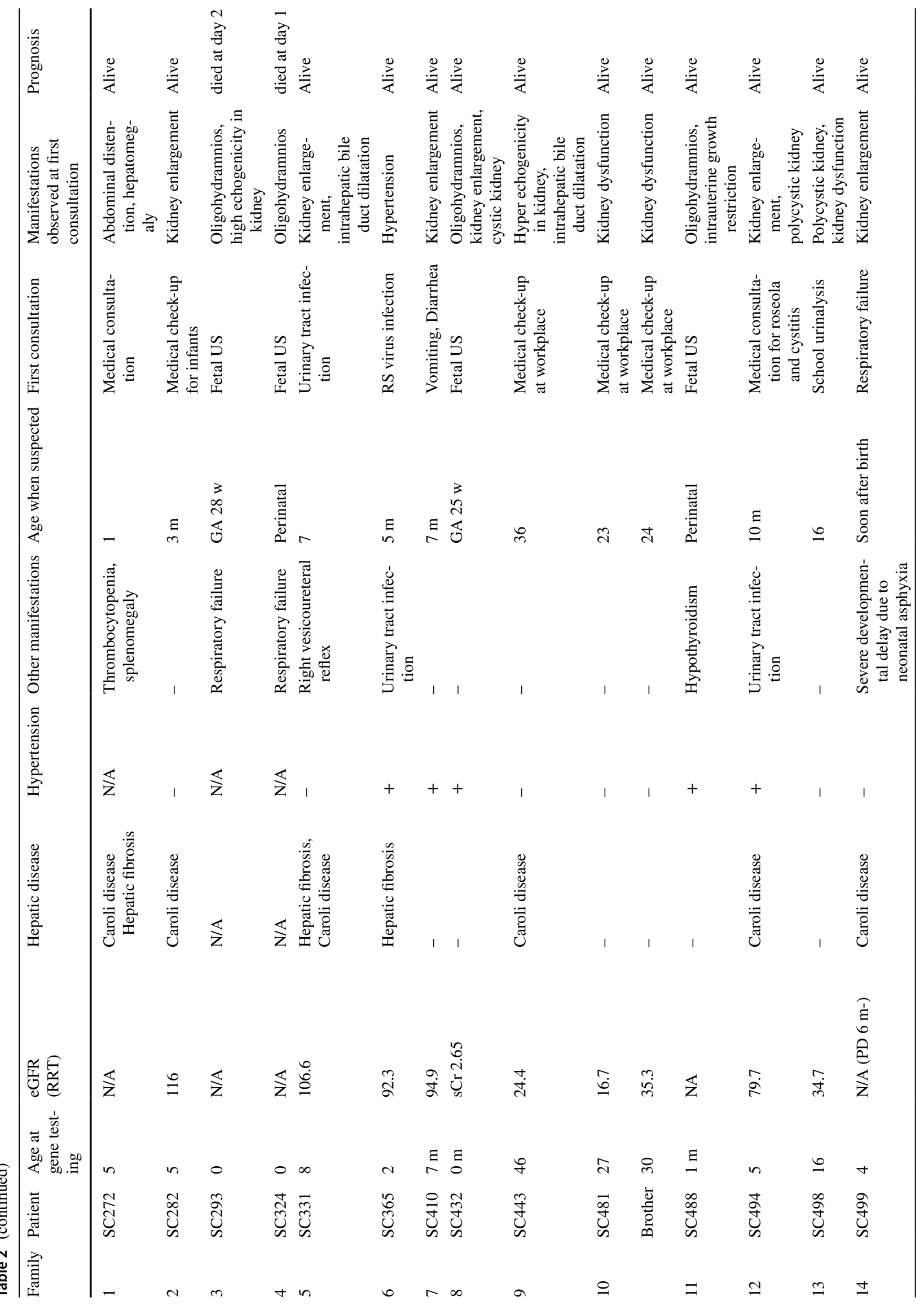




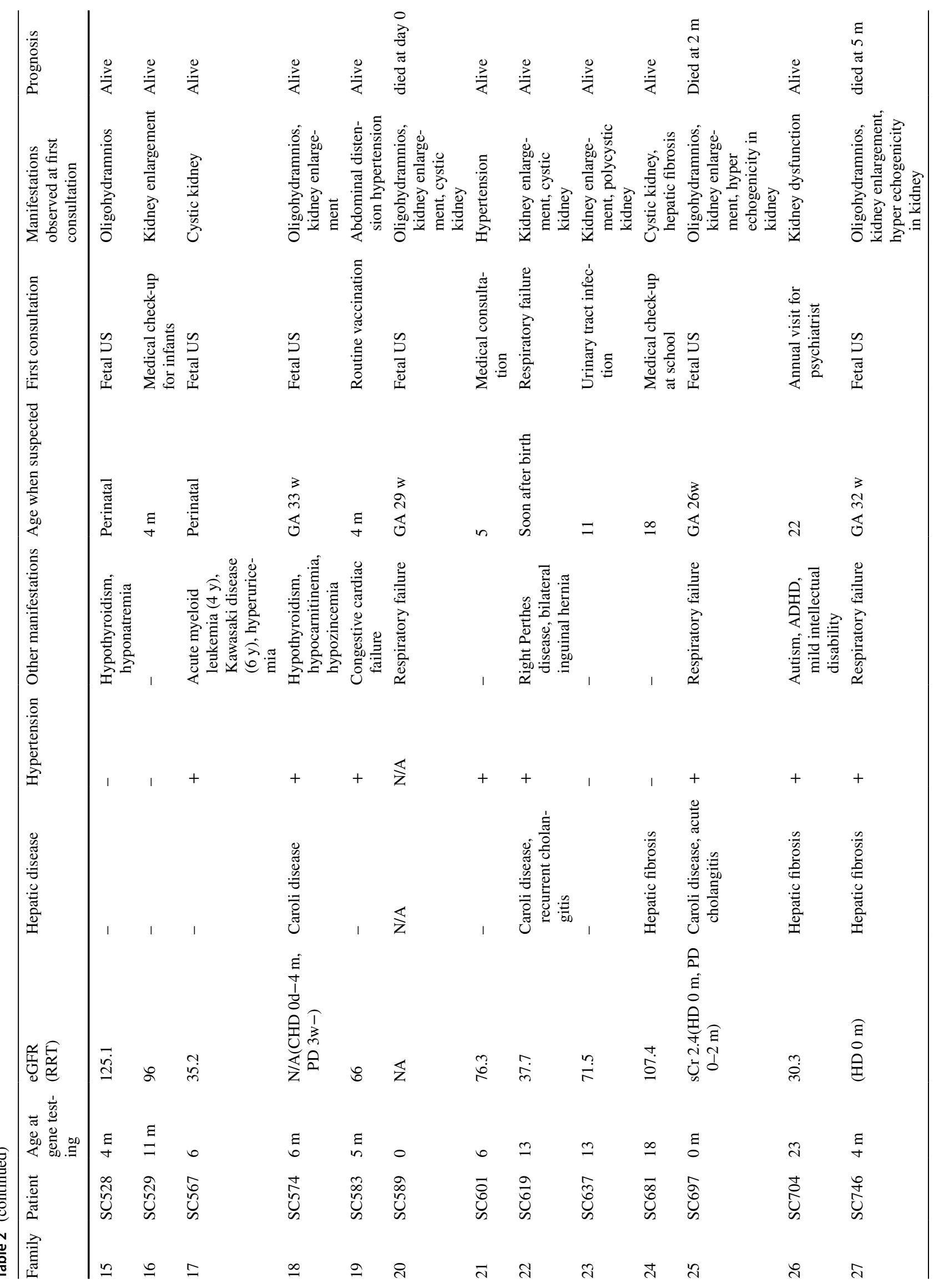




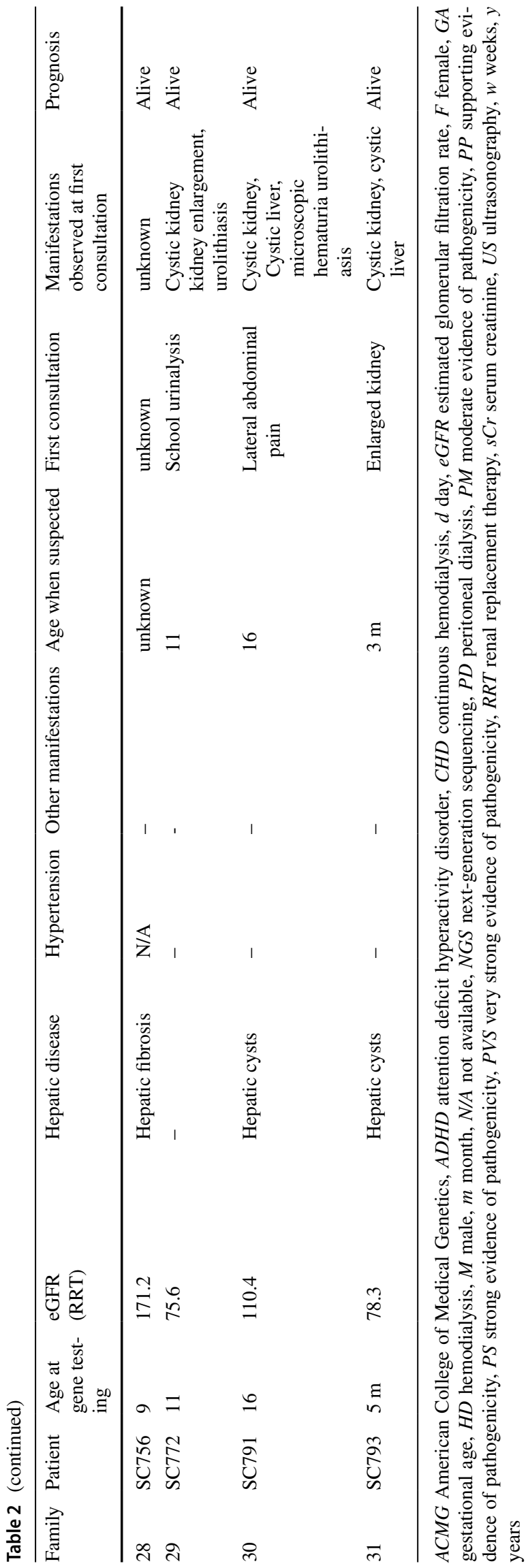

\section{Functional analysis via minigene assay}

Assays using the minigene system were performed using gDNA fragments from four patients (SC293, SC324, SC499, and SC589). The electrophoresis results of minigene transcripts for each mutation are shown in Fig. 2. Additionally, the in silico analysis and clinical course of each patient are shown in Table 3. The inserted sequences for each case are shown in Supplementary Fig. 2, and direct sequencing of the minigene transcript of each mutation is shown in Supplementary Fig. 3. For both mutations in SC499, which had no missense mutation (c.2713C > T, c. $6808+1 \mathrm{G}>\mathrm{A}$ ), both minigenes expressed a transcript that skipped an exon, in which the number of base pairs was a multiple of three, accompanied by multiple transcripts similar to the wild type transcript size, in smaller amounts. On the other hand, for SC293, SC324, and SC589, with one missense mutation associated with perinatal demise, every minigene expressed a normal transcript similar to the wild type. Additionally, for the splice site mutation (c.8555-2A > C) in SC 324, each minigene expressed a transcript that skipped exon 55 .

\section{Discussion}

This is the first multicenter report of genetically diagnosed ARPKD in the Japanese population. Although large-scale studies on ARPKD have been conducted in Europe and North America [7-10, 14], no study has been reported from Japan. In this study, 6 cases $(19.4 \%)$ were suspected to have ARPKD prenatally while the others were suspected or diagnosed after birth in various situations, mostly incidentally, which is in accordance with previous studies reporting that postnatal accidental finding is the most common reason for the initial visit [7, 8]. Screening urinalysis at school is performed every year in Japan, and this system enabled the diagnosis of ARPKD in two patients (SC498 and SC772). Almost half of the patients at the analyzed visit showed kidney functions within CKD stages 1 or 2 while four patients underwent renal replacement therapy. This finding suggests that the surviving patients show various phenotypes in the kidney, as previously described [9]. With the improvement of prognosis and advancement of renal insufficiency management, hepatobiliary disease is likely to become more prevalent. Caroli disease was observed in almost one-third of the patients, which is in accordance with previous reports [8, 10, 15]. In two cases of cholangitis, one patient underwent liver transplantation because of recurrent episodes, and another infant died due to suspected cholangitis at the age of two months. 


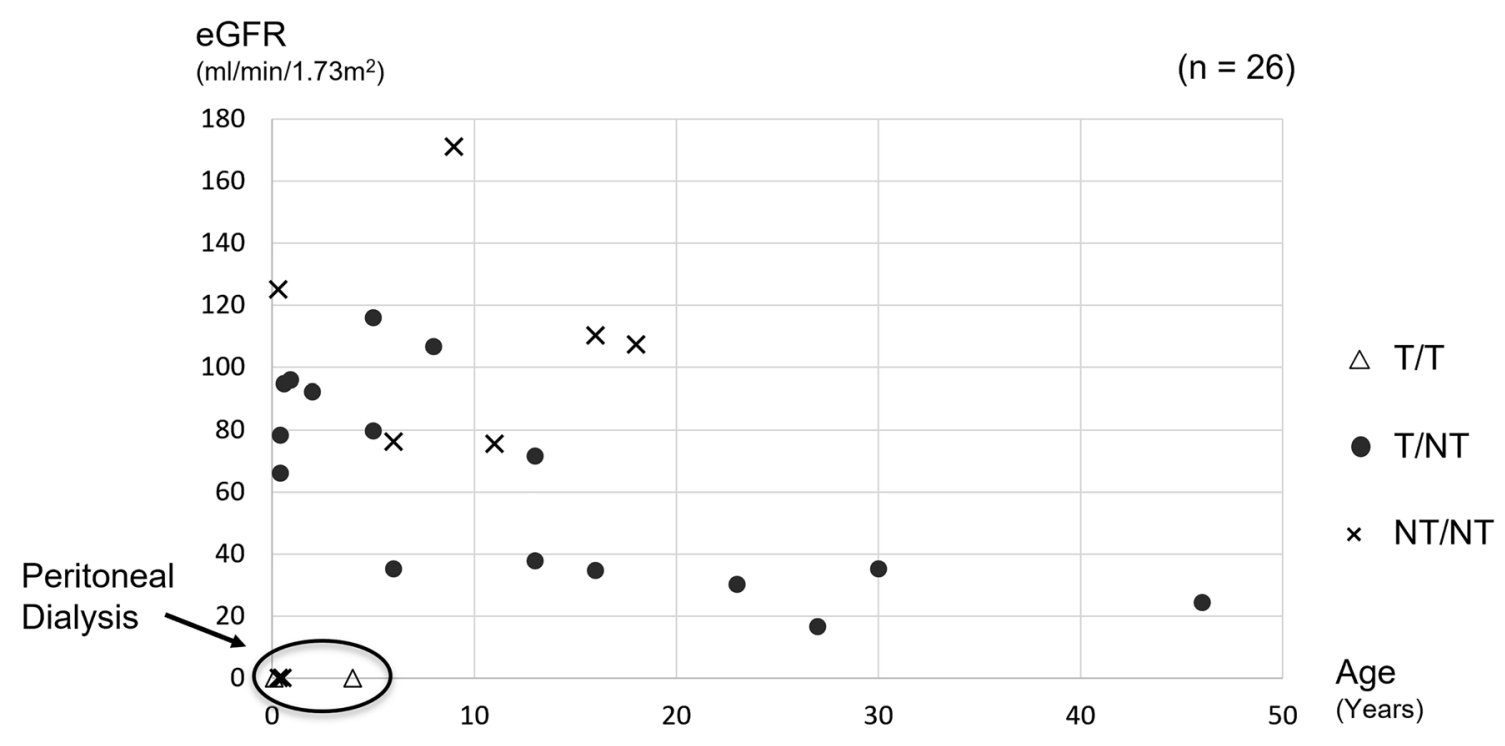

Fig. 1 The table shows eGFR of each case with the available data. Kidney functions varied among pediatric patients, and four patients underwent peritoneal dialysis while adult patients showed severe kidney dysfunction. Two patients with truncating mutations in both alleles underwent peritoneal dialysis. Six of eight patients with

According to previous reports, up to $80 \%$ of the children suffered from systemic hypertension $[9,10]$. In our study, almost $50 \%$ of the pediatric patients showed systemic hypertension, and among two of them hypertension was the first manifestation. Thus, it is essential to consider ARPKD as a differential diagnosis while examining pediatric patients with hypertension.

Congenital hypothyroidism was detected in three patients (SC488, SC528, and SC574) via newborn mass screening. To the best of our knowledge, only one case of ARPKD with congenital hypothyroidism has been reported [16]. In Japan, nearly $100 \%$ of newborns undergo mass screening, and the incidence of congenital hypothyroidism is approximately 1/4000 [17]. Considering the incidence of both diseases, it is unlikely that these two diseases co-occurred incidentally. Elevation in thyroid-stimulating hormone levels was observed in all three patients; thus, the patients were diagnosed with primary congenital hypothyroidism rather than central congenital hypothyroidism. Primary congenital hypothyroidism is traditionally subdivided into thyroid dysgenesis and dyshormonogenesis [18]. Dyshormonogenesis was presumed to be the cause of hypothyroidism in three patients because normal ultrasonography findings of the thyroid were observed. ARPKD is a cilia-related disease, and polyductin/fibrocystin localizes in the primary cilia and basal bodies of the cell. Primary cilia have been found in the thyrocytes of humans [19], and a direct relationship between ciliogenesis and thyroid follicle activity has been revealed in the functional pathology of the thyroid gland [20]. RNA two missense mutations were at CKD stage 1 or 2 , but two of them needed renal replacement therapy from a young age. $T$ truncating mutation, $N / T$ non-truncating mutation, $C K D$ chronic kidney disease, $e G F R$ estimated glomerular filtration rate

expression of the PKHDl gene in the thyroid gland was low but detectable; therefore, we assumed that the dysfunctions of primary cilia due to $P K H D 1$ gene mutation might lead to decreased follicular activity in the thyroid, which resulted in congenital hypothyroidism. More studies on primary cilia are needed to confirm the relationship between PKHDl and congenital hypothyroidism. Additionally, we need to carefully observe whether levothyroxine treatment of patients can be discontinued in the future.

In this study, we conducted a minigene assay to evaluate the splicing of $P K H D 1$. Studies in large-scale cohorts revealed genotype-phenotype correlations for $P K H D 1$; two truncating mutations display a severe phenotype associated with perinatal or neonatal death, and at least one missense mutation has been thought to be indispensable for survival during the neonatal period $[9,14]$. However, the SC499 patient survived the neonatal period, and did not need renal replacement therapy until the age of six months, although she did not harbor a missense mutation. Only a few reports have described milder cases with no missense mutations $[21,22]$. Both the nonsense and splice site variants in the minigene assay mainly expressed a transcript that skipped an exon with a multiple of three, resulting in in-frame mutations in both alleles. It has been suggested that nonsensemediated mRNA decay (NMD) may play a role in defining the phenotype of patients with ARPKD [21]. It can be speculated that exon skipping in exons 25 and 41, which leads to in-frame mutations, may lead to the circumvention of NMD and contribute to neonatal survival. Additionally, an 
(a) SC499-1 PKHD1 c.2713C>T p.GIn905Ter (Exon 25)

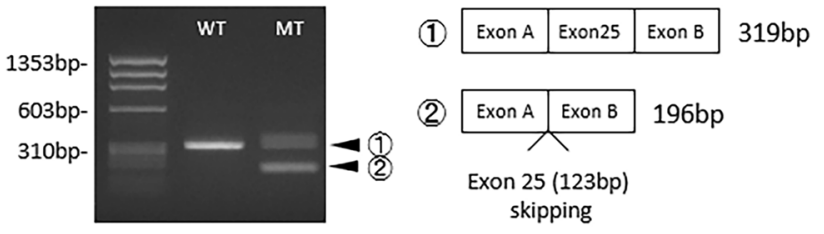

(c) SC293-2 PKHD1 c.9533G>T p.Gly3178Val (Exon 58)

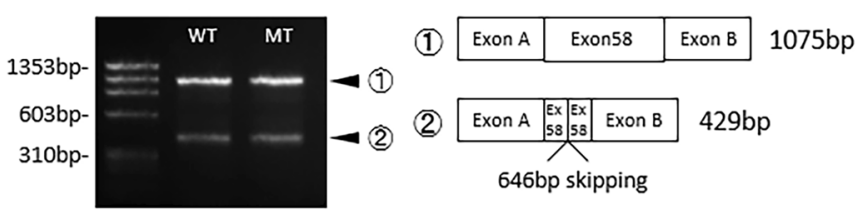

(e) SC324-2 PKHD1 c.8555-2A>C (Intron 54)

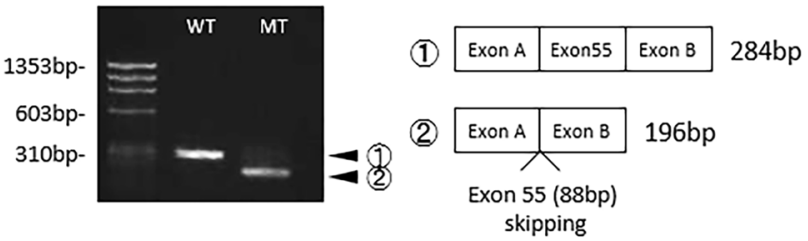

Fig. 2 Reverse transcription-polymerase chain reaction amplified products of minigene transcripts. a c.2713C $>\mathrm{T}$ (SC499-1) minigene expressed a full-length transcript in WT and a transcript that skipped exon 25 in MT. b c. $6808+1 \mathrm{G}>\mathrm{A}$ (SC499-2) minigene expressed a full-length transcript in WT, and a transcript that skipped exon 41 in MT. c c.9533G > T (SC293-2) minigene mainly expressed a fulllength transcript, and a few transcripts exhibiting $646 \mathrm{bp}$ deletion in exon 58 in both WT and MT. d c.3944 T> G (SC324-1) minigene

appropriate management during the neonatal period might have contributed to patient survival. Our results highlight the importance of functional analysis. Moreover, we call into question the fundamental belief that at least one missense mutation is necessary for survival through the perinatal or neonatal period.

On the other hand, three patients (SC293, SC324, and SC589) suffered from neonatal demise, and all of them had one missense mutation. One missense mutation does not guarantee perinatal and neonatal survival [14], and some studies have reported that missense mutations alter a splice enhancer motif that disrupts exon splicing, leading to aberrant $P K H D 1$ splicing [11]. We conducted a minigene assay for three cases to elucidate the mechanism involved in splicing that led to a severe phenotype. Nonsense mutations were detected in another allele of SC293 and SC589, and splice-site mutations were detected in SC324, which (b) SC499-2 PKHD1 c.6808+1G>A (IVS 41)

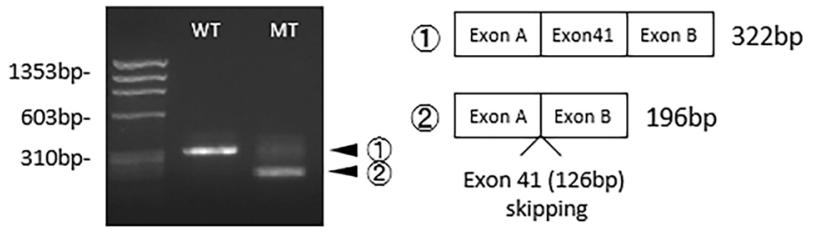

(d) SC324-1 PKHD1 c.3944T>G p.Leu1315Arg (Exon32)

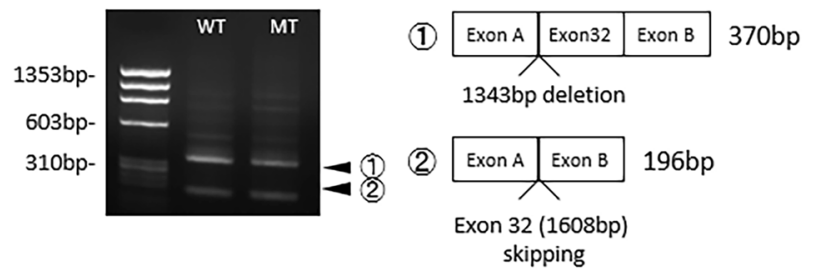

(f) SC589-1 PKHD1 c.983G>A p.Arg328GIn (Exon 14)

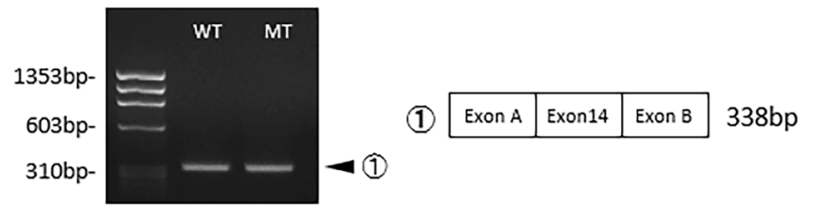

mainly expressed a transcript exhibiting 1343 bp deletion in exon 32 , exon 32 skipping, and multiple thin bands that could not be sequenced in both WT and MT. e c.8555-2A >C (SC324-2) minigene expressed a full-length transcript in WT, and a transcript exhibiting exon 55 skipping in MT. f) c.983G $>$ A (SC589-2) minigene expressed a full-length transcript in both WT and MT. WT, wild type. MT mutant

expressed a transcript that skipped exon 55 (88 bp), resulting in a truncating mutation. However, the minigene assay revealed that each missense mutation did not affect splicing. In SC324-1, a transcript with full-length exon 32 was not observed, but the splicing pattern was the same as that of the wild type; thus, we concluded that this mutation did not affect splicing. This is one of the limitations of minigene assays. Bergmann et al. demonstrated that the phenotypes due to PKHD1 mutations cannot be explained on the basis of the genotype alone, but may also depend on the background of other genes, epigenetic factors, and environmental influences [9, 14]. Our minigene assay results support this idea. Although the lack of mutational hotspots and variety phenotypes in PKHDl hampers further analysis for genotype-phenotype correlations, more studies are needed to investigate the mechanism involved in severe phenotypes. 
Table 3 Results of minigene assay and in silico analysis, and clinical course of patients with mutations conducted for minigene assay

\begin{tabular}{|c|c|c|c|c|c|c|c|}
\hline Case & cDNA & Amino acid & Exons & Mutation & $\begin{array}{l}\text { Results of minigene } \\
\text { assay }\end{array}$ & $\begin{array}{l}\text { In silico analysis by } \\
\text { human splicing Finder }\end{array}$ & Clinical course \\
\hline SC499-1 & c. $2713 \mathrm{C}>\mathrm{T}$ & p.Gln905Ter & 25 & Nonsense & $\begin{array}{l}\text { Exon } 25 \text { skipping } \\
\text { (123 bp) }\end{array}$ & $\begin{array}{l}\text { No significant impact on } \\
\text { splicing signals }\end{array}$ & \multirow{2}{*}{$\begin{array}{l}\text { Incubation after } \\
\text { birth. Extubation } \\
\text { at } 5 \text { months. Right } \\
\text { nephrectomy at } \\
6 \text { months, and follow- } \\
\text { ing this, peritoneal } \\
\text { dialysis was initiated. } \\
\text { Left nephrectomy at } \\
1 \text { year and } 4 \text { months }\end{array}$} \\
\hline SC499-2 & c. $6808+1 \mathrm{G}>\mathrm{A}$ & - & IVS 41 & Splice site & $\begin{array}{l}\text { Exon } 41 \text { skipping } \\
\text { (126 bp) }\end{array}$ & $\begin{array}{l}\text { Alteration of the WT } \\
\text { donor site, most prob- } \\
\text { ably affecting splicing }\end{array}$ & \\
\hline SC293-1 & c. $7113 \mathrm{~T}>\mathrm{G}$ & p.Tyr2371Ter & 45 & Nonsense & - & $\begin{array}{l}\text { Significant alteration of } \\
\text { ESE/ESS motifs ratio } \\
\text { Activation of a cryptic } \\
\text { acceptor site. Potential } \\
\text { alteration of splicing }\end{array}$ & $\begin{array}{l}\text { Died due to respiratory } \\
\text { failure at day } 2\end{array}$ \\
\hline SC293-2 & c. $9533 \mathrm{G}>\mathrm{T}$ & p.Gly3178Val & 58 & Missense & $\begin{array}{l}\text { Same transcript as wild } \\
\text { type }\end{array}$ & $\begin{array}{l}\text { Significant alteration of } \\
\text { ESE/ESS motifs ratio. } \\
\text { Activation of a cryptic } \\
\text { acceptor site. Potential } \\
\text { alteration of splicing }\end{array}$ & \\
\hline SC324-1 & c. $3944 \mathrm{~T}>\mathrm{G}$ & p.Leu1315Arg & 32 & Missense & $\begin{array}{l}\text { Same transcript as wild } \\
\text { type }\end{array}$ & $\begin{array}{l}\text { No significant impact on } \\
\text { splicing signals }\end{array}$ & $\begin{array}{l}\text { Died due to respiratory } \\
\text { failure at day } 2\end{array}$ \\
\hline SC324-2 & c. $8555-2 \mathrm{~A}>\mathrm{C}$ & - & IVS 54 & Splice site & $\begin{array}{l}\text { Exon } 55 \text { skipping } \\
\text { (88 bp) }\end{array}$ & $\begin{array}{l}\text { Alteration of the WT } \\
\text { acceptor site, most } \\
\text { probably affecting } \\
\text { splicing }\end{array}$ & \\
\hline SC589-1 & c. $983 \mathrm{G}>\mathrm{A}$ & p.Arg328Gln & 14 & Missense & $\begin{array}{l}\text { Same transcript as wild } \\
\text { type }\end{array}$ & $\begin{array}{l}\text { No significant impact on } \\
\text { splicing signals }\end{array}$ & $\begin{array}{l}\text { Died due to respiratory } \\
\text { failure at day } 0\end{array}$ \\
\hline SC589-2 & c. $8011 \mathrm{C}>\mathrm{T}$ & p.Arg2671Ter & 50 & Nonsense & - & $\begin{array}{l}\text { Significant alteration of } \\
\text { ESE/ESS motifs ratio }\end{array}$ & \\
\hline
\end{tabular}

ESE exonic splicing enhancer, ESS exonic splicing silencer, $W T$ wild type

This study had some limitations. This analysis was based on cases referred to our institute for gene testing from hospitals in Japan, not a nationwide registry in Japan. Therefore, the number of included patients was relatively small, and we could not obtain patient information with a long followup period. Not all patients were able to undergo parental analysis for clinical reasons; therefore, some "uncertain significance" variants were included in this study (Supplementary Table 8). In addition, in vivo analyses, such as RNA sequencing, were not performed for the evaluation of alternative splicing because sufficient and high-quality RNA samples could not be obtained. As mentioned, in the minigene assay, a normal splicing pattern was not observed in wild-type SC324-1.

In conclusion, this is the first multicenter report of genetically diagnosed ARPKD in the Japanese population. We detected 20 novel mutations in PKHD1. Clinical manifestations ranged from cases that died in the neonatal period to those incidentally found in adulthood. The complication of congenital hypothyroidism might be associated with dyshormonogenesis in the thyroid due to PKHDl gene mutations. From the minigene assay, we propose the importance of functional analysis, and call into question the fundamental belief that at least one missense mutation is necessary for survival during the perinatal or neonatal period.

Supplementary Information The online version contains supplementary material available at https://doi.org/10.1007/s10157-021-02135-3.

Acknowledgements The authors thank all patients, their social guardians, and primary doctors. We are profoundly grateful to Mrs. Yoshimi Nozu, Ms. Yuko Noguchi, Mrs. Tetsuko Yamanouchi, and Mrs. Ming Juan Ye (Kobe University) for their excellent technical assistance. Data for patients with SC574 mutations have been published elsewhere in Japanese journals. We would like to thank Editage (www.editage.com) for English language editing. The following doctors provided patient samples for the study: Kyoko Takano (Shinshu University), Shinichi Shiona (Oita Prefectural Hospital), Eriko Nishi and Nobuhiko Okamoto (Osaka Women's and Children's Hospital), Mai Sato and Koichi Kamei (National Center for Child Health and Development), Shinsuke Matsumoto (Matsudo City Hospital), Kenichiro Kobayashi (Amagasaki General Medical Center), Yoshinobu Nagaoka (Sapporo Medical University), Chiharu Kinoshita (Kyoto Min-iren chuo Hospital), Kengo Kajihara (Kumamoto Medical Center), Yasufumi Ohtsuka (Saga University Hospital), Shoichiro Kanda (The university of Tokyo Hospital), Ryutaro Hisatomi and Rika Fujimaru (Osaka City General Hospital), Taichi Kitaoka (Osaka University Hospital), Mina Takeuchi and Yoshitsugu Kaku (Fukuoka Children's Hospital), Mari Kurokawa 
(Kyushu University Hospital), Seiichi Tomotaki and Tatsuya Okamoto (Kyoto University Hospital), Akio Yamada (Nishichita General Hospital), Daisuke Ogino (Yamagata University Hospital), Masashi Nishida (University Hospital, Kyoto Prefectural University of Medicine), Ikuyo Narita (Hirosaki University Hospital), Atsushi Tashiro (JCHO Chukyo Hospital), Shiro Iwawaki (JCHO Gunma Central Hospital), and Hiroshi Tamura (Kumamoto University).

Funding This work was supported by the Health Labor Sciences Research Grant for the Research on Measures for Intractable Diseases (H24-nanchi-ippan-041 to K.I.; H29-nanchi-ippan-039 to N.M.) and Japan Society for the Promotion of Science (KAKENHI Grant Numbers JP15K09261 and 18K08243 to N.M.). All procedures performed in studies involving human participants were conducted in accordance with the ethical standards of the Institutional Review Board of Kobe University Graduate School of Medicine (IRB approval numbers 65 and 301) and the 1964 Helsinki Declaration and its later amendments or comparable ethical standards. Comprehensive informed consent was obtained from all individual participants in the study and/or their guardians regarding the use of patients' clinical data.

\section{Declarations}

Conflict of interest K.I. has received grant support from Daiichi Sankyo Co., Ltd. and Zenyaku Kogyo Co., Ltd. M. M discloses being employed by Kobe Gakuin University, which received funding from KNC Laboratories Inc. (Kobe, Japan). The author further discloses being a scientific adviser for Daiichi-Sankyo Co. (Tokyo, Japan) and JCR Pharma Co. (Ashiya, Japan).

Open Access This article is licensed under a Creative Commons Attribution 4.0 International License, which permits use, sharing, adaptation, distribution and reproduction in any medium or format, as long as you give appropriate credit to the original author(s) and the source, provide a link to the Creative Commons licence, and indicate if changes were made. The images or other third party material in this article are included in the article's Creative Commons licence, unless indicated otherwise in a credit line to the material. If material is not included in the article's Creative Commons licence and your intended use is not permitted by statutory regulation or exceeds the permitted use, you will need to obtain permission directly from the copyright holder. To view a copy of this licence, visit http://creativecommons.org/licenses/by/4.0/.

\section{References}

1. Ward CJ, Hogan MC, Rossetti S, Walker D, Sneddon T, Wang $\mathrm{X}$, et al. The gene mutated in autosomal recessive polycystic kidney disease encodes a large, receptor-like protein. Nat Genet. 2002;30(3):259-69. https://doi.org/10.1038/ng833.

2. Onuchic LF, Furu L, Nagasawa Y, Hou X, Eggermann T, Ren $\mathrm{Z}$, et al. PKHD1, the polycystic kidney and hepatic disease 1 gene, encodes a novel large protein containing multiple immunoglobulin-like plexin-transcription-factor domains and parallel beta-helix 1 repeats. Am J Hum Genet. 2002;70(5):1305-17. https://doi.org/10.1086/340448.

3. Follit JA, Li L, Vucica Y, Pazour GJ. The cytoplasmic tail of fibrocystin contains a ciliary targeting sequence. J Cell Biol. 2010;188(1):21-8. https://doi.org/10.1083/jcb.200910096.

4. Lu H, Galeano MCR, Ott E, Kaeslin G, Kausalya PJ, Kramer $\mathrm{C}$, et al. Mutations in DZIP1L, which encodes a ciliary-transition-zone protein, cause autosomal recessive polycystic kidney disease. Nat Genet. 2017;49(7):1025-34. https://doi.org/10. 1038/ng.3871.

5. Sweeney WE, Avner ED, et al. Polycystic Kidney Disease, Autosomal Recessive. In: Adam MP, Ardinger HH, Pagon RA, et al., editors. Gene Reviews. Seattle: University of Washington; 2001.

6. Buscher R, Buscher AK, Weber S, Mohr J, Hegen B, Vester U, et al. Clinical manifestations of autosomal recessive polycystic kidney disease (ARPKD): kidney-related and non-kidneyrelated phenotypes. Pediatr Nephrol. 2014;29(10):1915-25. https://doi.org/10.1007/s00467-013-2634-1.

7. Burgmaier K, Kilian S, Bammens B, Benzing T, Billing $\mathrm{H}$, Buscher A, et al. Clinical courses and complications of young adults with autosomal recessive polycystic kidney disease (ARPKD). Sci Rep. 2019;9(1):7919. https://doi.org/10.1038/ s41598-019-43488-w.

8. Adeva M, El-Youssef M, Rossetti S, Kamath PS, Kubly V, Consugar MB, et al. Clinical and molecular characterization defines a broadened spectrum of autosomal recessive polycystic kidney disease (ARPKD). Medicine (Baltimore). 2006;85(1):1-21. https://doi.org/10.1097/01.md.0000200165.90373.9a.

9. Bergmann C, Senderek J, Windelen E, Kupper F, Middeldorf I, Schneider F, et al. Clinical consequences of PKHD1 mutations in 164 patients with autosomal-recessive polycystic kidney disease (ARPKD). Kidney Int. 2005;67(3):829-48. https://doi. org/10.1111/j.1523-1755.2005.00148.x.

10. Guay-Woodford LM, Desmond RA. Autosomal recessive polycystic kidney disease: the clinical experience in North America. Pediatrics. 2003;111(5 Pt 1):1072-80. https://doi.org/10.1542/ peds.111.5.1072.

11. Boddu R, Yang C, O'Connor AK, Hendrickson RC, Boone B, Cui $\mathrm{X}$, et al. Intragenic motifs regulate the transcriptional complexity of Pkhd1/PKHD1. J Mol Med (Berl). 2014;92(10):104556. https://doi.org/10.1007/s00109-014-1185-7.

12. Nagano C, Nozu K, Morisada N, Yazawa M, Ichikawa D, Numasawa $\mathrm{K}$, et al. Detection of copy number variations by pair analysis using next-generation sequencing data in inherited kidney diseases. Clin Exp Nephrol. 2018;22(4):881-8. https://doi.org/ 10.1007/s10157-018-1534-x.

13. Nozu K, Iijima K, Kawai K, Nozu Y, Nishida A, Takeshima $\mathrm{Y}$, et al. In vivo and in vitro splicing assay of SLC12A1 in an antenatal salt-losing tubulopathy patient with an intronic mutation. Hum Genet. 2009;126(4):533-8. https://doi.org/10.1007/ s00439-009-0697-7.

14. Bergmann C, Senderek J, Sedlacek B, Pegiazoglou I, Puglia P, Eggermann T, et al. Spectrum of mutations in the gene for autosomal recessive polycystic kidney disease (ARPKD/PKHD1). J Am Soc Nephrol. 2003;14(1):76-89. https://doi.org/10.1097/ 01.asn.0000039578.55705.6e.

15. Turkbey B, Ocak I, Daryanani K, Font-Montgomery E, Lukose L, Bryant J, et al. Autosomal recessive polycystic kidney disease and congenital hepatic fibrosis (ARPKD/CHF). Pediatr Radiol. 2009;39(2):100-11. https://doi.org/10.1007/ s00247-008-1064-X.

16. Jeha GS, Tatevian N, Heptulla RA. Congenital hypothyroidism in association with Caroli's disease and autosomal recessive polycystic kidney disease: patient report. J Pediatr Endocrinol Metab. 2005;18(3):315-8. https://doi.org/10.1515/jpem.2005. 18.3.315.

17. Mass Screening Committee, Japanese Society for Pediatric Endocrinology, Japanese Society for Mass Screening, Nagasaki K, Minamitani K, Anzo M, et al. Guidelines for Mass Screening of Congenital Hypothyroidism (2014 revision). Clin Pediatr 
Endocrinol. 2015;24(3):107-33. https://doi.org/10.1297/cpe.24. 107.

18. Peters C, van Trotsenburg ASP, Schoenmakers N. Diagnosis of endocrine disease: congenital hypothyroidism: update and perspectives. Eur J Endocrinol. 2018;179(6):R297-317. https:// doi.org/10.1530/EJE-18-0383.

19. Martin A, Hedinger C, Haberlin-Jakob M, Walt H. Structure and motility of primary cilia in the follicular epithelium of the human thyroid. Virchows Arch B Cell Pathol Incl Mol Pathol. 1988;55(3):159-66. https://doi.org/10.1007/BF02896572.

20. Fernandez-Santos JM, Utrilla JC, Vazquez-Roman V, VillarRodriguez JL, Gutierrez-Aviles L, Martin-Lacave I. Primary Cilium in the human thyrocyte: changes in frequency and length in relation to the functional pathology of the thyroid gland. Thyroid. 2019;29(4):595-606. https://doi.org/10.1089/thy.2018. 0401.
21. Frank V, Zerres K, Bergmann C. Transcriptional complexity in autosomal recessive polycystic kidney disease. Clin J Am Soc Nephrol. 2014;9(10):1729-36. https://doi.org/10.2215/CJN. 00920114.

22. Szabo T, Orosz P, Balogh E, Javorszky E, Mattyus I, Bereczki C, et al. Comprehensive genetic testing in children with a clinical diagnosis of ARPKD identifies phenocopies. Pediatr Nephrol. 2018;33(10):1713-21. https://doi.org/10.1007/ s00467-018-3992-5.

Publisher's Note Springer Nature remains neutral with regard to jurisdictional claims in published maps and institutional affiliations. 